\title{
Domain of Attraction of $\alpha$-stable Distributions under Finite Mixture Models ${ }^{1}$
}

C.E.G. OTINIANO², Departamento de Estatística, UnB, 70910-900 Brasília, DF, Brazil.

C.R. GONÇALVES 3 , Departamento de Matemática, UnB, 70910-900 Brasília, DF, Brazil.

\begin{abstract}
In this work, we study the asymptotic distribution of the normalized sum of independent, identically distributed random variables under the finite mixture models. In the Theorem we give necessary conditions for a distribution function of a mixed population with $\mathrm{k}$ components to belong to the domain of attraction of an $\alpha$-stable distribution, by assuming that each component of the mixture also pertains to the domain of attraction of an $\alpha$-stable distribution. Examples are given to illustrate the result.
\end{abstract}

Keywords. Mixed population, domain of attraction, tail index, stable distribution.

\section{Introduction}

Finite mixtures have practical applications in several areas. The books [2], [9],[6] and [5] describe finite mixture models, properties and applications. Analogously, [9] exhibit examples of direct applications of finite mixture models for fishing, economics, medicine, psychology, paleontology, botany, agriculture, zoology and reliability, among others. The indirect applications include outliers, mixture of normal for testing robustness, cluster analysis, latent structure models, bayesian inference, nonparametric estimation of densities, approximation of mixture models with nonmixture models. Finally, [7] describes multivariate survival models which use mixture of positive stable distributions.

On the last three decades, data from heavy tails distributions have been observed in several areas. Whenever the observed quantities can be approximated by sums of small terms and the data empirical densities exhibit heavy tails and asymmetry, the Central Limit Theorem together with the strong empirical evidence, indicate that a more realistic model might be obtained with distributions in a $\alpha$-stable domain attraction. Generalized extreme value (GEV) is an example of such distributions, as well as [8] proposes a computational procedure to estimate their distributions.

\footnotetext{
${ }^{1}$ The authors were partially supported by PROCAD/CAPES, FINATEC/UnB, PRONEX/ FAPDF and CASADINHO/CAPES.

${ }^{2}$ cira@unb.br

${ }^{3}$ catia@mat.unb.br
}

Recebido em 04 Setembro 2009; Aceito em 17 Maio 2010. 
Given $Z_{1}, \ldots Z_{n}, Z$ random variables (r.v.'s) independent and identically distributed (iid) with common distribution $G$, the r.v. $Z$ (or $G$ ) is called $\alpha$-stable, with $0<\alpha \leq 2$, if there are constants $D_{n} \in \mathbf{R}$ such that

$$
S_{n}=Z_{1}+\ldots+Z_{n} \stackrel{d}{=} n^{1 / \alpha} Z+D_{n} .
$$

Does not have closed formula for $G$, but its corresponding characteristic function is well known and given by

$$
\varphi(t)=\left\{\begin{array}{rll}
\exp \left\{i \mu t-\sigma^{\alpha}|t|^{\alpha}\left[1-i \beta \operatorname{sign}(t) \tan \left(\alpha \frac{\pi}{2}\right)\right]\right\}, & \text { if } & \alpha \neq 1 \\
\exp \left\{i \mu t-\sigma|t|\left[1-i \beta \frac{2}{\pi} \operatorname{sign}(t) \ln (t)\right]\right\}, & \text { if } & \alpha=1,
\end{array}\right.
$$

where $0<\alpha \leq 2$ (stability index), $-1 \leq \beta \leq 1$ ( symmetry parameter), $\sigma>0$ (scale parameter) and $\mu \in \mathbf{R}$ (location parameter) uniquely characterizes the distribution $G$, except for $\alpha=2$. In this case we denote $G$ by $S_{\alpha}(\sigma, \beta, \mu)$.

From (1.1) we have that the distribution of $Z, G$, is a distribution of normalized sums, such as, $\frac{S_{n}-D_{n}}{n^{1 / \alpha}} \stackrel{d}{=} Z$, suggesting that $G$ can be obtained as an asymptotic limit distribution of normalized sums of iid va's, known as Generalized Central Limit Theorem. According to [4], this result can be written in terms of domain of attraction as follows:

Let $X, X_{1}, X_{2}, \ldots$ r.v.'s i.i.d. with common distribution $F$. The r.v. $X$ or $F$ belongs to the domain of attraction of a $\alpha$-stable, $Z_{\alpha} \stackrel{d}{=} G_{\alpha} \quad\left(F \in D\left(G_{\alpha}\right)\right)$, if there are constants $b_{n} \in \mathbf{R}$ such that

$$
\frac{S_{n}-b_{n}}{n^{1 / \alpha} L(n)} \stackrel{d}{\rightarrow} Z_{\alpha}
$$

where " $\stackrel{d}{\longrightarrow}$ " denotes convergence in distribution, $S_{n}=X_{1}+X_{2}+\cdots+X_{n}$,

$$
b_{n}= \begin{cases}\mu, & \text { if } 1<\alpha \leq 2 \\ 0, & \text { if } \quad 0<\alpha<1 \\ 0, & \text { if } \quad \alpha=1 \text { and F symmetric }\end{cases}
$$

and $L$ is a slowly varying function, that is, $L(t x) \sim L(t)$. We use the notation $g(y) \sim h(y)$ to indicate that $\lim _{y \rightarrow \infty} \frac{g(y)}{h(y)}=1$.

For $0<\alpha<2$, an important characterization of the domain of attraction of a $\alpha$-stable distribution, which can be found in [3] or [1], is as follows:

$$
F \in D\left(G_{\alpha}\right) \Leftrightarrow F(-x) \sim C^{I} x^{-\alpha} L(x) \text { and } \bar{F}(x)=1-F(x) \sim C^{D} x^{-\alpha} L(x),
$$

where $C^{I} \geq 0$ and $C^{D} \geq 0$ are such that $C^{I}+C^{D}>0$ and $L$ is a slowly varying function.

In this article, we will extend the result above, for the case where the original distribution of basic variables is a finite mixture of $k$ components

$$
F(x)=\sum_{i=1}^{k} p_{i} F_{i}(x), \quad \text { with } \quad p_{i} \geq 0 \quad \text { and } \quad \sum_{i=1}^{k} p_{i}=1,
$$


where the $i$ th component (distribution function of the $i$ th sub-population) $F_{i} \in$ $D\left(G_{\alpha_{i}}\right)$.

\section{Domain of Attraction of $\alpha$-stable Distributions based on Random Samples of Mixed Populations}

Along this section, we present, for a distribution function $F$ as in (1.3), necessary conditions to belong to the domain of attraction of an $\alpha$-stable distribution, To accomplish it we use a random sample of a mixed population of $k$ components, where each component $F_{i}$ is in the domain of attraction of an $\alpha$-stable distribution. In other words, the following theorem sets up necessary conditions to describe the limit distribution of normalized sum of $n$ i.i.d. random variables, where the common distribution is a mixture of $k$ components whose tails decrease as the power $x^{-\alpha_{i}}, i=1, \ldots, k$.

To do so, firstly observe that by $(1.2)$, if $F_{i} \in D\left(G_{\alpha_{i}}\right)$ for $i=1, \ldots, k$, then

$$
F_{i}(-x) \sim C_{i}^{I} x^{-\alpha_{i}} L_{i}(x) \text { and } \bar{F}_{i}(x)=1-F_{i}(x) \sim C_{i}^{D} x^{-\alpha_{i}} L_{i}(x),
$$

where $C_{i}^{I} \geq 0$ and $C_{i}^{D} \geq 0$ is such that $C_{i}^{I}+C_{i}^{D}>0$ and $L_{i}$ is slowly varying function, for $i=1, \ldots, k$.

Theorem 2.1. Let $X_{1}, X_{2}, \ldots, X_{n}$ r.v.'s i.i.d. with a common distribution function $F$ given by (1.3). If $F_{i} \in D\left(G_{\alpha_{i}}\right)$ for $i=1, \ldots, k$, then

(a) $F \in D\left(G_{\alpha_{m}}\right) \quad$ with $\quad \alpha_{m}=\min \left\{\alpha_{1}, \ldots, \alpha_{k}\right\}$, if $\alpha_{i} \neq \alpha_{j}$ for all $i \neq j$;

(b) $F \in D\left(G_{\alpha}\right) \quad$ with the constants (2.1) satisfying $\frac{C_{1}^{I}}{C_{1}^{D}}=\frac{C_{2}^{I}}{C_{2}^{D}}=\cdots=\frac{C_{n}^{I}}{C_{n}^{D}}$, if $\alpha=\alpha_{1}=\cdots=\alpha_{k}$.

Proof. (a) From (1.3) we have

$$
F(-x)=\sum_{i=1}^{k} p_{i} F_{i}(-x), \quad \bar{F}(x)=\sum_{i=1}^{k} p_{i} \bar{F}_{i}(x) .
$$

Using (2.1) in (2.2), we obtain

$$
F(-x) \sim \sum_{i=1}^{k} p_{i} C_{i}^{I} x^{-\alpha_{i}} L_{i}(x) \quad \text { and } \quad \bar{F}(x) \sim \sum_{i=1}^{k} p_{i} C_{i}^{D} x^{-\alpha_{i}} L_{i}(x)
$$

Since $\alpha_{i} \neq \alpha_{j}$ for all $i \neq j$, let $\alpha_{m}=\min \left\{\alpha_{1}, \ldots, \alpha_{k}\right\}$. Then, on account of the $L_{i}^{\prime} s$ being slowly varying and $\lim _{x \rightarrow \infty} x^{\alpha_{m}-\alpha_{i}}=0$ for all $i \neq m$, the expressions (2.3) turn out to be

$$
\begin{aligned}
F(-x) & \sim x^{-\alpha_{m}} \sum_{i=1}^{k} p_{i} C_{i}^{I} x^{\alpha_{m}-\alpha_{i}} L_{i}(x) \\
& \sim p_{m} C_{m}^{I} x^{-\alpha_{m}} L_{m}(x)
\end{aligned}
$$


and

$$
\begin{aligned}
\bar{F}(x) & \sim x^{-\alpha_{m}} \sum_{i=1}^{k} p_{i} C_{i}^{D} x^{\alpha_{m}-\alpha_{i}} L_{i}(x) \\
& \sim p_{m} C_{m}^{D} x^{-\alpha_{m}} L_{m}(x) .
\end{aligned}
$$

Thus (1.2) is satisfied for the component with lower tail index, and (a) follows.

(b) If $\alpha=\alpha_{1}=\cdots=\alpha_{k}$, the expressions (2.3) can be written as

$$
F(-x) \sim x^{-\alpha} \sum_{i=1}^{k} p_{i} C_{i}^{I} L_{i}(x) \quad \text { and } \quad \bar{F}(x) \sim x^{-\alpha} \sum_{i=1}^{k} p_{i} C_{i}^{D} L_{i}(x) .
$$

Using the fact that the sum of slowly varying functions is a slowly varying function and the condition $\frac{C_{1}^{I}}{C_{1}^{D}}=\frac{C_{2}^{I}}{C_{2}^{D}}=\cdots=\frac{C_{n}^{I}}{C_{n}^{D}}$, we have

$$
F(-x) \sim \frac{A}{C_{1}^{D} C_{2}^{D} \ldots C_{k}^{D}} x^{-\alpha} \sum_{i=1}^{k} p_{i} C_{i}^{D} L_{i}(x) \text { and } \bar{F}(x) \sim x^{-\alpha} \sum_{i=1}^{k} p_{i} C_{i}^{D} L_{i}(x)
$$

because

$$
\begin{aligned}
\sum_{i=1}^{k} p_{i} C_{i}^{I} L_{i}(x)= & \frac{1}{C_{1}^{D} C_{2}^{D} \ldots C_{k}^{D}}\left[C_{1}^{I} C_{2}^{D} \ldots C_{k}^{D}\left(p_{1} C_{1}^{D} L_{1}(x)\right)\right. \\
& \left.+\cdots+C_{k}^{I} C_{1}^{D} C_{2}^{D} \ldots C_{k-1}^{D}\left(p_{k} C_{k}^{D} L_{k}(x)\right)\right] \\
= & \frac{A}{C_{1}^{D} C_{2}^{D} \ldots C_{k}^{D}} \sum_{i=1}^{k} p_{i} C_{i}^{D} L_{i}(x)
\end{aligned}
$$

where

$$
A=C_{1}^{I} C_{2}^{D} \ldots C_{k}^{D}=C_{2}^{I} C_{1}^{D} C_{3}^{D} \ldots C_{k}^{D}=\cdots=C_{k}^{I} C_{1}^{D} C_{2}^{D} \ldots C_{k-1}^{D} .
$$

Taking the slowly varying function $L(x)=\sum_{i=1}^{k} p_{i} C_{i}^{D} L_{i}(x), \quad C^{I}=\frac{A}{C_{1}^{D} C_{2}^{D} \ldots C_{k}^{D}}$ and $C^{D}=1$, in (2.5), the proof of (b) follows from (1.2).

Remark. The result above is also valid for the Gaussian distribution which is the only $\alpha$-stable distribution with light tail. That is, if the components $F_{i}, i=1, \ldots, k$ of the mixture

$$
F(x)=\sum_{i=1}^{k} p_{i} F_{i}(x)
$$

belong to the domain of attraction of Gaussian $\mathcal{N}\left(\mu, \sigma^{2} / 2\right)=S_{2}(\sigma, 0, \mu), F_{i} \in$ $D(2), \quad i=1, \ldots, k$, then $F \in D(2)$. 
For proof, as in [1], consider the following characterization,

$$
F_{i} \in D(2) \Leftrightarrow 1-F_{i}(x)+F_{i}\left(-x^{-}\right)=\mathrm{o}\left(h_{i}(x)\right), \quad i=1, \ldots, k,
$$

where o $(h(y))=g(y)$ denotes the limit $\lim _{y \rightarrow \infty} \frac{g(y)}{h(y)}=0$, and $h_{i}(x)$ is a slowly varying function $h_{i}(x)=\int_{|y| \leq x} y^{2} d F_{i}(x)$, which is the truncated second moment of $F_{i}$.

Since

$$
\begin{aligned}
1-F(x)+F\left(-x^{-}\right) & =1+\sum_{i=1}^{k} p_{i}\left[-F_{i}(x)+F_{i}\left(-x^{-}\right)\right] \\
& =\sum_{i=1}^{k} p_{i}\left[1-F_{i}(x)+F_{i}\left(-x^{-}\right)\right]
\end{aligned}
$$

and the product of slowly varying functions is a slowly varying function, from (2.6) and (2.7) it follows that

$$
1-F(x)+F\left(-x^{-}\right)=\mathrm{o}(H(x)),
$$

with $H(x)=h_{1}(x) \cdot h_{1}(x) \ldots h_{k}(x)$. Hence $F \in D(2)$.

Example 2.1. Let $X_{1}, X_{2}, \ldots, X_{n}$ be a random sample of a mixed population whose distribution function is $F(x)=p F_{1}(x)+(1-p) F_{2}(x)$, where $0<p<1$ and $F_{1}, F_{2}$ are the stable distributions

$$
F_{1}=S_{\alpha_{1}}\left(\sigma_{1}, \beta_{1}, \mu_{1}\right) \text { and } F_{2}=S_{\alpha_{2}}\left(\sigma_{2}, \beta_{2}, \mu_{2}\right),
$$

with $0<\alpha_{1}, \alpha_{1} \leq 2$. We have

$$
F_{1} \in D\left(\alpha_{1}\right) \text { and } F_{2} \in D\left(\alpha_{2}\right)
$$

because, by [10] and [8] among others, the tails of such distributions satisfy

$$
F_{i}(-x) \sim C_{i}^{I} x^{-\alpha_{i}} \quad \text { and } \quad \bar{F}_{i}(x) \sim C_{i}^{D} x^{-\alpha_{i}}, \quad i=1,2,
$$

where

$$
C_{i}^{I}=\frac{\left(1-\alpha_{i}\right)\left(1-\beta_{i}\right) \sigma_{i}^{\alpha_{i}}}{2 \Gamma\left(2-\alpha_{i}\right) \cos \left(\frac{\pi \alpha_{i}}{2}\right)}, \quad C_{i}^{D}=\frac{\left(1-\alpha_{i}\right)\left(1+\beta_{i}\right) \sigma_{i}^{\alpha_{i}}}{2 \Gamma\left(2-\alpha_{i}\right) \cos \left(\frac{\pi \alpha_{i}}{2}\right)} \quad \text { for } \quad \alpha_{i} \neq 1
$$

and

$$
C_{i}^{I}=\frac{\left(1-\beta_{i}\right) \sigma_{i}^{\alpha_{i}}}{\pi}, \quad C_{i}^{D}=\frac{\left(1+\beta_{i}\right) \sigma_{i}^{\alpha_{i}}}{\pi} \quad \text { for } \quad \alpha_{i}=1 .
$$

If $\alpha_{1}<\alpha_{2}$ then $F(-x) \sim p C_{1}^{I} x^{-\alpha_{1}}$ and $\bar{F}(x) \sim p C_{1}^{D} x^{-\alpha_{1}}$. Therefore $F_{1} \in D\left(\alpha_{1}\right)$, which illustrates (a) of the Theorem.

If $\alpha=\alpha_{1}=\alpha_{2}$ and $\beta_{1} \beta_{2}+2\left(\beta_{1} \beta_{2}\right)=0$, we have

$$
F(-x) \sim \frac{1-\beta_{1}}{1+\beta_{1}}\left[p C_{1}^{I}+(1-p) C_{2}^{I}\right] x^{-\alpha} \quad \text { and } \quad \bar{F}(x) \sim\left[p C_{1}^{I}+(1-p) C_{2}^{I}\right] x^{-\alpha} \text {. }
$$


Then $F \in D(\alpha)$, which illustrates (b) of the Theorem.

Example 2.2. Let $X_{1}, X_{2}, \ldots, X_{n}$ a random sample of a mixed population whose distribution function is $F(x)=p \mathcal{G}_{\gamma_{1}}(x)+(1-p) \mathcal{G}_{\gamma_{2}}(x)$, where $0<p<1, \gamma_{1}>$ $1 / 2, \gamma_{2}<-1 / 2$ and $\mathcal{G}_{\gamma_{1}}, \mathcal{G}_{\gamma_{2}}$ are the following generalized extreme value distributions (GEV's)

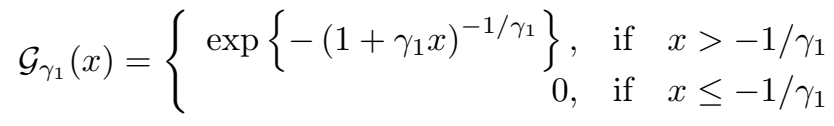

and

$$
\mathcal{G}_{\gamma_{2}}(x)=\left\{\begin{array}{rlc}
\exp \left\{-\left(1+\gamma_{2} x\right)^{-1 / \gamma_{2}}\right\}, & \text { if } & x>-1 / \gamma_{2} \\
0, & \text { if } & x \leq-1 / \gamma_{2} .
\end{array}\right.
$$

Note that $\mathcal{G}_{\gamma_{1}} \in D\left(G_{1 / \gamma_{1}}\right)$ and $\mathcal{G}_{\gamma_{2}} \in D\left(G_{-1 / \gamma_{2}}\right)$. Since

$$
\lim _{t \rightarrow \infty} \frac{\overline{\mathcal{G}}_{\gamma_{1}}(t x)}{\overline{\mathcal{G}}_{\gamma_{1}}(t)}=x^{-1 / \gamma_{1}}, x>-1 / \gamma_{1},
$$

there are a slowly varying function $L_{1}$ such that $\overline{\mathcal{G}}_{\gamma_{1}}(t x) \sim x^{-1 / \gamma_{1}} L_{1}(x)$ (see, for example [1]).That is, (1.2) is valid with

$$
\mathcal{G}_{\gamma_{1}}(-x) \sim 0 . x^{-1 / \gamma_{1}} L_{1}(x) \text { and } \overline{\mathcal{G}}_{\gamma_{1}}(x) \sim 1 . x^{-1 / \gamma_{1}} L_{1}(x) .
$$

Similarly for $\mathcal{G}_{\gamma_{2}}$ we can verify that there is a slowly varying function $L_{2}$ such that

$$
\mathcal{G}_{\gamma_{2}}(-x) \sim 0 . x^{1 / \gamma_{2}} L_{2}(x) \text { and } \overline{\mathcal{G}}_{\gamma_{2}}(x) \sim 1 . x^{1 / \gamma_{2}} L_{2}(x) .
$$

Thus, if $0<\frac{1}{\gamma_{1}}<\frac{-1}{\gamma_{2}}<2$, using (2.8) and (2.9) we have

$$
F(-x) \sim 0 \text { and } \bar{F}(x) \sim p x^{-1 / \gamma_{1}} L_{1}(x),
$$

because $\frac{1}{\gamma_{1}}+\frac{1}{\gamma_{2}}>0$. Therefore $F \in D\left(G_{1 / \gamma_{1}}\right)$.

Finally, if $0<\alpha=\frac{1}{\gamma_{1}}=\frac{-1}{\gamma_{2}}<2$, using (2.8) and (2.9) it follows that

$$
F(-x) \sim 0 \text { and } \bar{F}(x) \sim p x^{-\alpha} L_{1}(x) .
$$

Then $F \in D\left(G_{\alpha}\right)$.

\section{Numerical Illustration}

To illustrate the example 2 we consider $\gamma_{1}=0.6, \gamma_{2}=-1$ and $p=0.8$. In Figure 1 , the graph on the left shows the density of $\mathcal{G}_{\gamma_{1}}$ and $\mathcal{G}_{\gamma_{2}}$, the graph on the right shows the mixture of densities

$$
f(x)=p \mathcal{G}_{\gamma_{1}}(x)+(1-p) \mathcal{G}_{\gamma_{2}}(x) .
$$



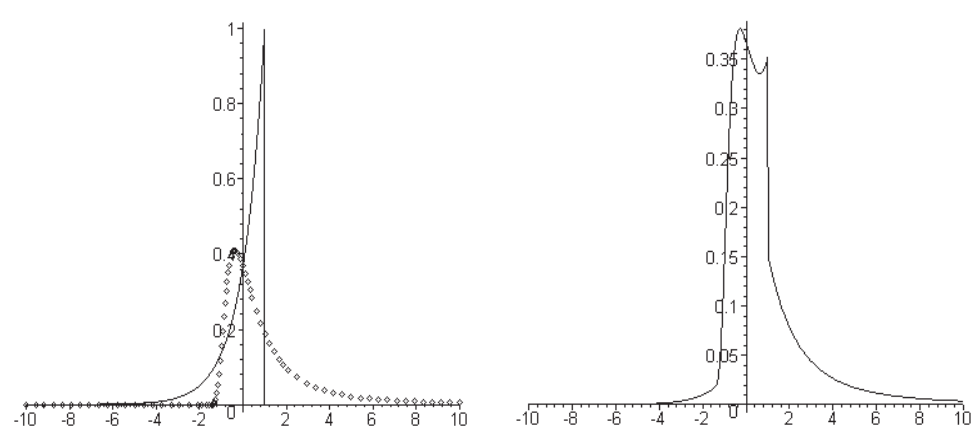

Figure 1: The graph on the left ${ }^{x}$ shows densities GEV. The graph ơ on the right shows the mixture of densities GEV.

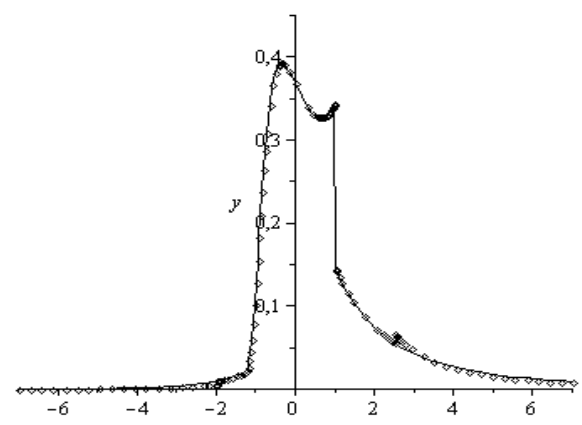

Figure 2: Density function (3.1) (continuous line) and estimated density function (points).

Random samples of the mixture were generated depending upon the following steps

(a) Two uniform random variables $u_{1}$ and $v_{2}$ with are generated with the program MAPLE.

(b) If $u_{1}<p$, we use $u_{2}$ to generate r.v. $x$ using $x=\mathcal{G}_{\gamma_{1}}^{-1}\left(u_{2}\right)=\left(\left(-\ln u_{2}\right)^{-\gamma_{1}}\right) / \gamma_{1}$.

(c) If $u_{2}<p$, we use $u_{2}$ to generate r.v. $x$ using $x=\mathcal{G}_{\gamma_{2}}^{-1}\left(u_{2}\right)$.

For to estimate the tails of the density of (3.1), we simulated 200 data and using the software Xtreme we obtained the estimates of the parameters of the stable distribution: $\hat{\alpha}=1.063, \hat{\beta}=0.095$ and $\hat{\sigma}=0.7377$. This confirms our results because in this case $\alpha=\min \left\{1 / \gamma_{1},-1 / \gamma_{2}\right\}=1$.

Figure 2 displays the density function (3.1) and the estimated density function.

\section{Conclusion}

The tails of the asymptotic distribution of the sum of normalized i.i.d. random variables, whose common distribution is a mixture of $k$ components with different 
tail indexes decreasing as the power $x^{-\alpha_{i}}, \quad i=1 \ldots, n$, decrease with the power of the lower tail index of the $k$ components. In the case of tail index of the components being equal, an additional condition between the weight of the tails of the components is necessary for the asymptotic distribution of the normalized sum decrease with the same power of the components.

\section{Acknowledgments}

The authors would like to thank the reviewers for their helpful comments.

Resumo. Neste trabalho estudamos a distribuição assintótica da soma normalizada de variáveis aleatórias independentes e identicamente distribuídas, sob modelos de mistura finita. No Teorema damos condições necessárias para a função de distribuição de de uma população mista com $k$ componentes estar no domínio de atração de uma distribuição $\alpha$ - estável, assumindo que cada componente da mistura também esta no domínio de atração de uma distribuição $\alpha$ - estável. Exemplos são dados para mostrar o resultado.

\section{References}

[1] P. Embrechts, T. Mikosch, C. Kluppelberg, "Modelling Extremal Events for Insurance and Finance", Springer - Verlag, Berlin, 1997.

[2] B.S. Everitt, D.J. Hand, "Finite Mixture Distributions", Chapman and Hall, London, 1981.

[3] W. Feller, "An Introduction to Probability Theory and its Applications II", 3rd edition, Wiley, New York, 1968.

[4] I.A. Ibragimov, Yu. V. Linnik, "Independent and Stationary Sequences of Random Variables", Wolters-Noordhoff, Groningen, 74-85, 1971.

[5] B. G. Lindsay, "Mixture Models: Theory, Geometry and Applications", Institute of Mathematical and Statistics, Hayward, CA, 1995.

[6] G.J. McLachlan, K.E.Basford, "Mixture Models: Aplications to Clustering", Marcel Dekker, New York, 1988.

[7] N. Ravishanker, D.K. Dey, Multivariate survival models with a mixture of positive stable frailties, Journal Methodology and Computing in Applied Probability, 2 (2004), 293-308.

[8] G. Samorodnitsky, M. Taqqu, "Stable Non-Gaussian Random Process", Chapman and Hall, New York, 1994.

[9] D.M. Titterington, A.F.M. Smith, U.E. Makov, "Statistical Analysis of Finite Mixture Distributions", Wiley, New York, 1985.

[10] V. M. Zolotarev, "One Dimensional Stable Distributions", American Mathematical Society, Providence, 1986. 